\title{
Participation in Free and Open Source Communities: An Empirical Study of Community Members' Perceptions.
}

\author{
Andrew Schofield ${ }^{\prime}$ and Professor Grahame S. Cooper ${ }^{2}$ \\ 1 Information Systems Institute, University of Salford, Salford, M5 4WT, \\ UK, a.j.schofield@pgt.salford.ac.uk, \\ WWW Home page: http://www.postgrad.isipartnership.net/ aschofield/ \\ 2 School of Computing, Science, and Engineering, University of Salford, \\ Salford, M5 4WT, UK, g.s.cooper@salford.ac.uk, \\ WWW Home page: \\ http://www.cse.salford.ac.uk/profiles/profile.php?profile=G.S.Cooper
}

\begin{abstract}
Although the defining factors of Free and Open Source Software (FOSS) are generally seen as the availability and accessibility of the source code, it is what these facilitate that is perhaps of more significance. Source code availability allows the sharing of code, skills, knowledge, and effort, focused on a particular piece of software under development. The result of this is the FOSS community, which although often perceived as a single group, is actually many small groups, each bound by a common interest in a particular piece of software and using the Internet as a communication medium. Although there have been studies focusing on the motivation of FOSS developers to contribute to software, there has been little investigation into the motives, attitudes, and the culture within the communities as a whole. There is much more to most of these communities than software development. Many also have extensive support networks for the use of software, portals for research, and social facilities. This paper describes the results of an investigation into how FOSS community members perceive the communities that they belong to, their reasons for being in the community, and the manner in which they participate.
\end{abstract}

\section{Introduction}

Free and Open Source Software communities remain elusive and intangible despite the significant amount of research that has been done on the subject. The significance of these communities is also something that has been under much debate. Some authors (Raymond, 2000; Lanzara \& Morner, 2003; Oh \& Jeon, 2004) describe FOSS communities as entirely virtual systems that operate almost exclusively over the Internet on a global scale. Other authors (Krishnamurthy, 2002; O'Mahony \& Ferraro, 2004) maintain that in many cases, a significant amount of FOSS communities often operates off-line in the 'real world', and that a considerable quantity of FOSS development is actually performed by individuals. It is probable that in actual fact, FOSS development is a mixture of both these theories. While some projects will have large numbers of people working on them, other projects may have few or a single developer. Furthermore, although some projects will exist entirely on-

Please use the following format when citing this chapter:

Schofield, A., and Cooper, G.S., 2006, in IFIP International Federation for Information Processing, Volume 203, Open Source Systems, eds. Damiani, E., Fitzgerald, B., Scacchi, W., Scotto, M., Succi, G., (Boston: Springer), pp. 221-231 
line, others may involve off-line meetings between people, especially between the core development team and in projects originating from within organisations (Schofield \& Mitra 2005).

The community members themselves are not easily put into categories. The work by Zhang \& Storck (2001) illustrates this issue by putting forward the definition of "peripheral members". These are members of the FOSS community that may not directly participate within the community. To take this idea further, the only visible members of a FOSS community are those who participate in discussion forums, bulletin boards, named code development, or those who make themselves known in other ways. Members who visit the on-line communities, perhaps reading from forums, but not posting anything, may still be considered to be part of the community but will remain unknown to other members. In contrast to this, it is the belief of many authors (Sagers, 2004; O'Mahony, 2004) that social interaction is the foundation to FOSS community existence, which suggests that without a critical mass of participating members, a community cannot exist.

How members interact with their community is ultimately defined by the available interaction mechanisms and the particular needs of the member. There are several reasons why people may choose to become part of a FOSS community. The bulk of the literature on this subject has focused on the motivation of developers (Hann et al 2004; Hertel et al 2003; Lakhani \& Wolf 2003; Scacchi et al 2005; Schofield \& Mitra 2004). Suggested reasons include; pragmatic reasons for needing specific software functionality, enjoyment of software development as a hobby, educational benefits, feelings of belonging to a community and/or to a large scale movement, the need for recognition, self-gratification from a sense of achievement, and career advancement though skill acquisition. Although the above work gives some insight into the reasons members have for being involved in community-based FOSS development, it does not provide a whole picture of motivation in FOSS communities beyond software development, nor how members' perception of the community defines their participation

\section{Research Method}

The data collected for this research used a predominantly quantitative on-line survey method. Reaching members of FOSS communities for data collection is inherently difficult, for the reasons of intangibility and levels of participation explained above. The sample set of this research consisted of a particular type of Open Source group within the UK, the Linux User Groups (LUGs). The term is slightly deceptive as most of these groups do not only concentrate on the Linux Operating System but on a wide variety of other Open Source operating systems, application and programs. The research findings presented in this paper are based on the 145 survey submissions received 
Although the survey was directed at the UK LUGs, it was open for others to participate. Analysis revealed that of the total number of submissions, approximately $12 \%$ came from people who were not part of a FOSS society, club, or user group. Many of the LUGs are involved in software development in some way, and members may also be involved in other software development communities. The survey used dealt with individuals' experiences of on-line FOSS communities in general, not specifically the LUGs, and although for some members, experience of a FOSS community will only be the LUG, others' will certainly have a broader experience including other communities. The survey results demonstrate this, as many members have referred to other communities in their submissions.

The survey itself dealt with several aspects of FOSS communities and the attitudes and participation of community members. This paper covers the areas of the survey that collected data about the specific reasons a member may have for participating, in terms of the actual activities involved, and how and for what purpose a member makes use of communities.

\section{Research Findings}

The basic motivation for anyone making use of an on-line FOSS community is to perform some function, i.e. to use an on-line tool to achieve a desired action. It is which functions a member uses and why they use them that the initial phase of the research attempted to discover. This section of the survey collected community members' perceptions of what they actually do within FOSS communities and the pragmatic reasons for participating. Research subjects were presented with several possible reasons for making use of on-line FOSS communities;

- To find out how to perform a task in a software application (Problem solving).

- To help other people to use software applications (Providing support).

- To suggest alterations or improvements to software programs (Peer review).

- To contribute bug fixes or code improvements (Software development).

- To meet people or talk to people with similar interests (Social exchange).

The survey question was designed to allow members to select more than one reason or to specify one or more of their own. Expressed as the actual number of choices, figure 1 shows how many members chose the above reasons i.e. 127 members chose (not exclusively) problem solving to be a reason for participating in a FOSS community. Figure 2 shows this data presented in percentage form (i.e. $25 \%$ of all the choices submitted by all members were for providing support).

As not all members of FOSS communities are developers, it was expected that the peer review, and software development factors would be less popular than those relating to support. In addition to these choices, members also posted other reasons including: being the leader/manager of a community, lurking (Members may have 
many reasons to lurk perhaps born out of a simple interest in observing discussion), to encourage the advocacy of FOSS, to build business relations, to learn industry standards and trends, and finally, just for fun!

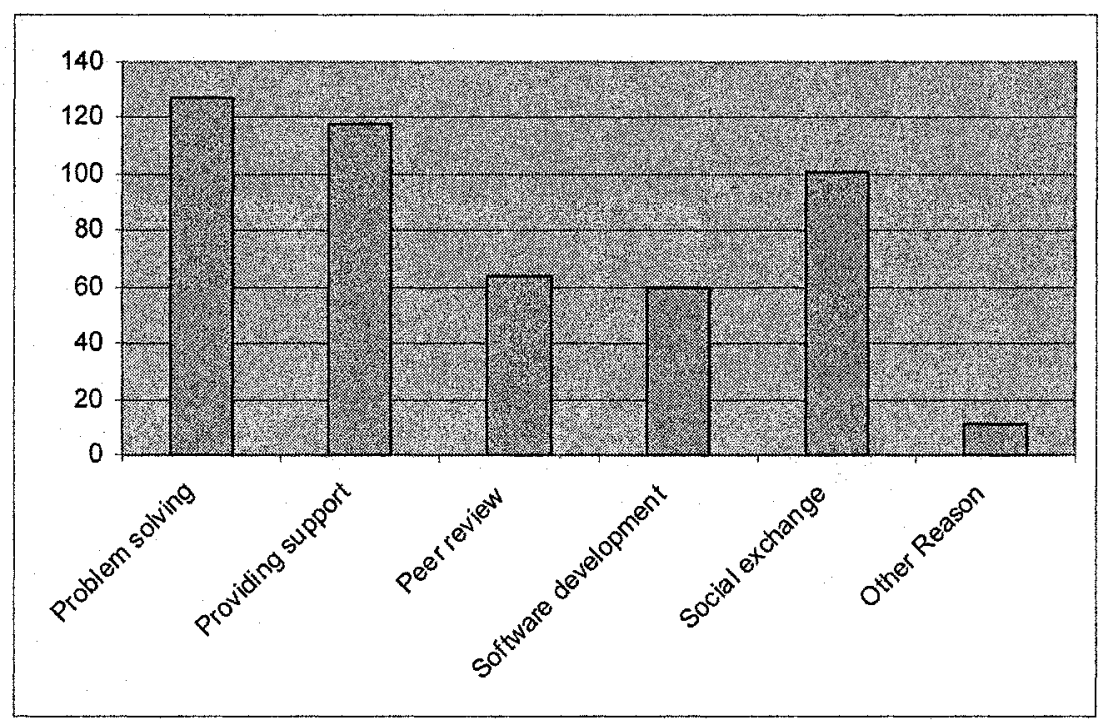

Figure 1: Reasons for Participation

The first phase of the research identified the reasons why community members participate in FOSS communities, in terms of what activities they are involved in. The next phase of the research was to investigate how these community activities are used,

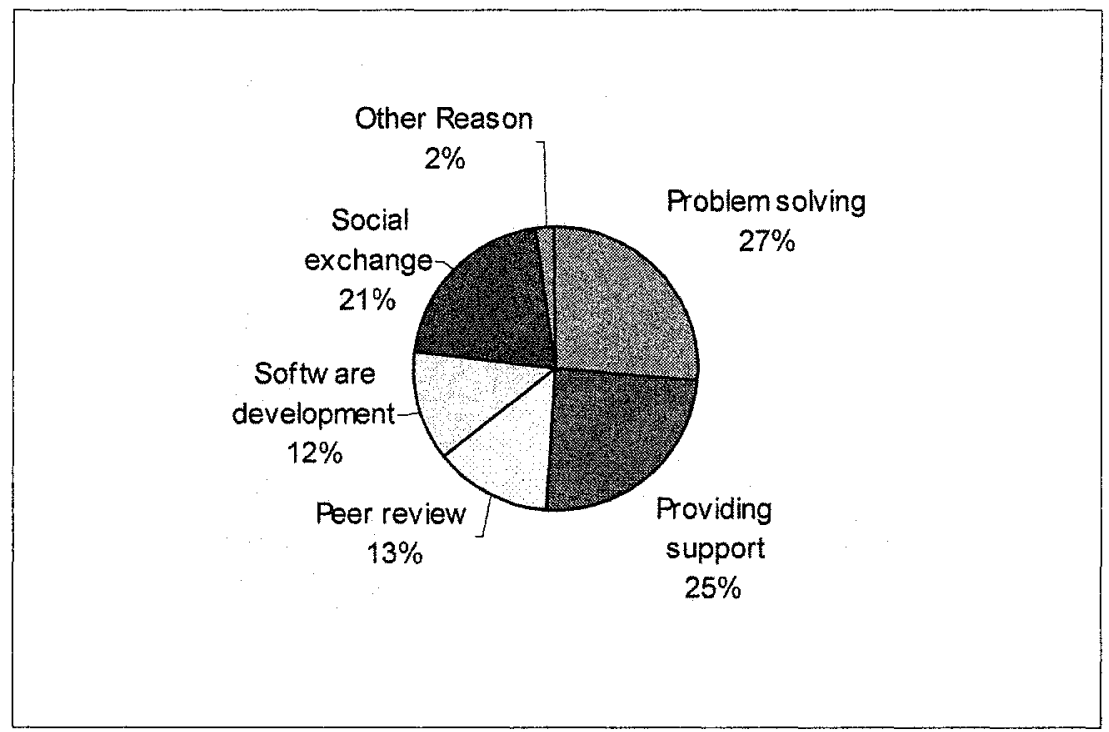

Figure 2: Reasons for Participation as a Percentage 
and to collect self-reflective perceptions of why they are used in the manner to which

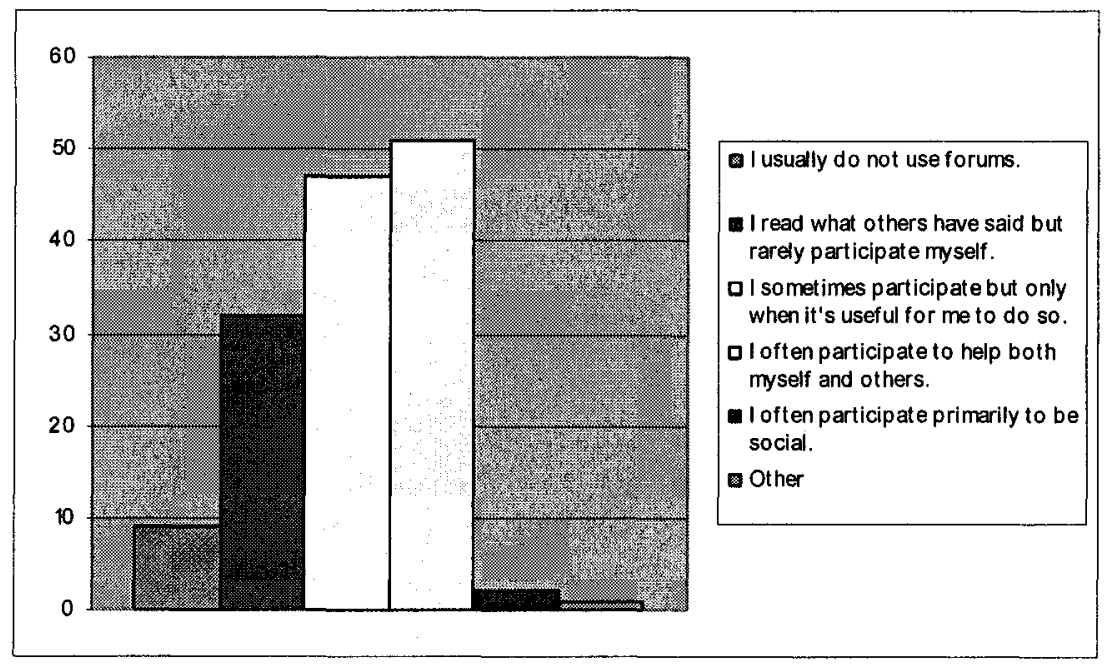

Figure 3: Use of Support Forums

the member refers. This phase of the research was split into two sections; the first looking exclusively at the community aspects which provide support for the use of software, and the second at the aspects revolving around software development.

The members were presented with the following alternative ways of interacting with FOSS support community forums:

- I usually do not use forums.

- I read what others have said but rarely participate myself.

- I sometimes participate but only when it's useful for me to do so.

- I often participate to help both myself and others.

- I often participate primarily to be social.

Many members chose to leave additional comments for this question, almost all of which stating that they preferred mailing lists to discussion boards. There was some suggestion that discussion boards were more for beginners, and that they are more focussed on specific issues as oppose to mailing lists which have more general coverage. The interface of the majority of discussion boards was also criticised and listed as another reason for members preferring mailing lists.

Finally, those members with software development experience were asked how they use FOSS community functions for software development. The following possibilities were given and, as before, members could specify their own alternatives.

- I mainly participate just to get help with my own development work. 
- I participate both to receive help myself with my own work and to help others with theirs.

- I mainly participate to get involved in the development projects of others.

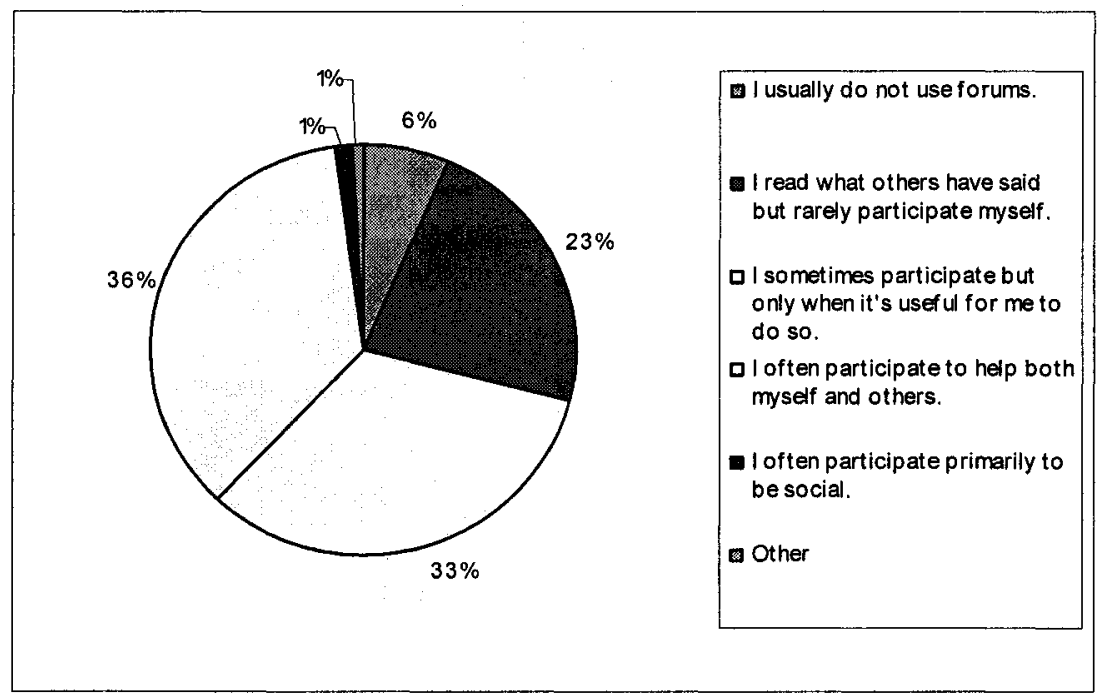

Figure 4: Reasons for Participation as a Percentage

- I mainly participate to be sociable.

The order of the questions in the survey and the request that the completion of this question is by developers only, is based on the assumption that all developers are also users of FOSS software. More specifically this means that both users and developers will make use of the support forums, but that only developers will make use of the software development forums. It is acknowledged that in some cases these may be the

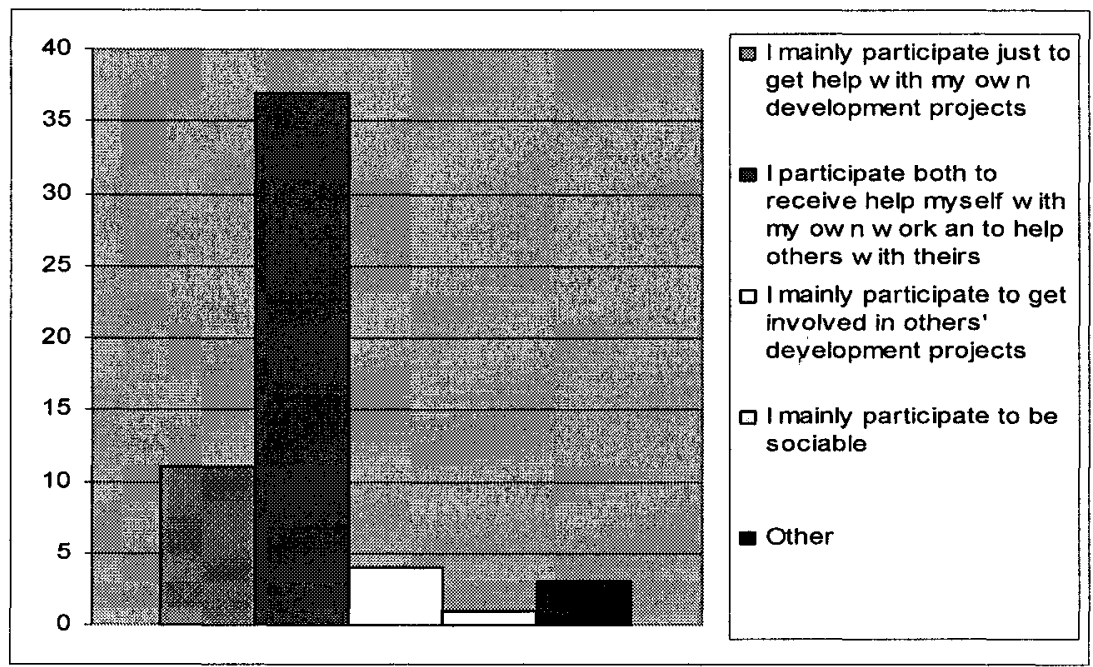

Figure 5: Use of Development Forums 
same forums but it is still possible to separate the two activities.

Again the members were also given the opportunity to provide their own answer to the question in case none of these options were appropriate. For this question members were asked to choose only one option from the list. Figure 3 shows the choices made by the members and Figure 4 the results as a percentage.

Figure 5 shows the results of this question being put to the developers and Figure 6 shows the same data in a percentage format.

Other uses specified by the developers were: to use the development forums as a source of research material, to disseminate software to others, to use FOSS development activities for personal professional development, and again, just for the fun of it.

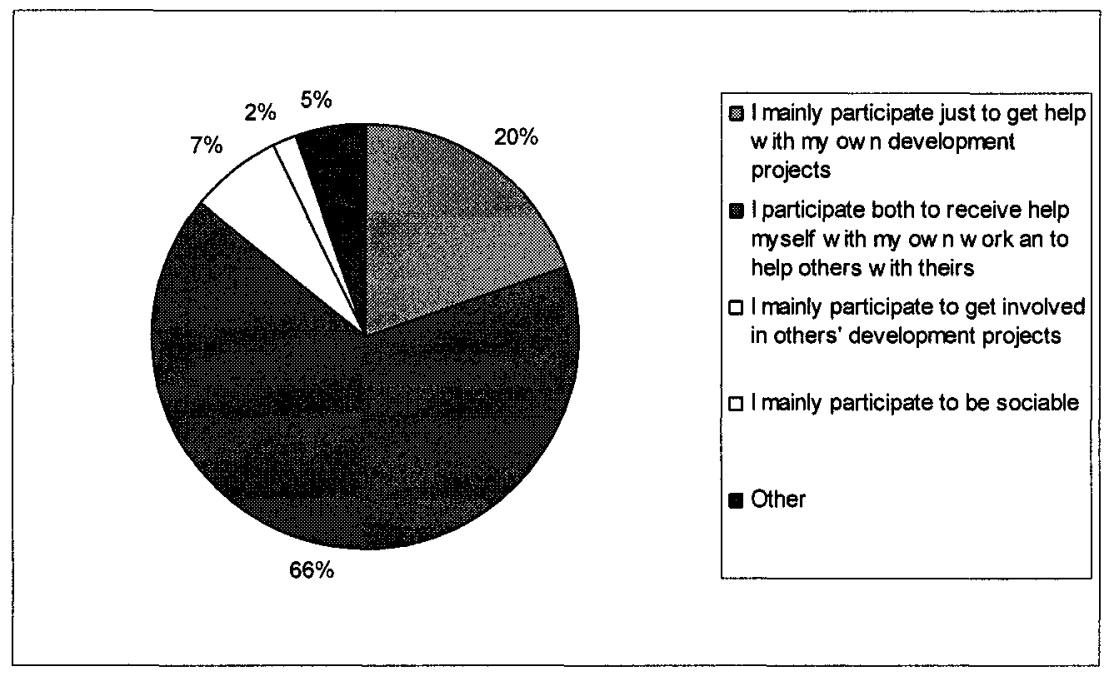

Figure 6: Use of Development Forums as a Percentage

\section{Research Analysis}

By their very nature, FOSS development and the communities performing it are open to anyone who wants to get involved at any level. The fact that they are also facilitated by the Internet means that a community is not usually confined by any geographical constraints, but rather exists on an international or global scale. It is this fact that justifies the use of the UK Linux/Open Source User groups as the sample set for this research. The groups may have members from all over the world and each member is likely to be involved with a myriad of other diverse communities. The collection of the data for this research itself is a good example. The request for 
participation was sent to specific UK groups and resulted in submissions arriving from many other countries which were not specifically targeted. An acknowledged potential limitation of the research is that LUGs are perhaps more likely to focus on support than other kinds of FOSS community. There are some communities that are almost entirely focused on software development and much less on support. Although many LUG members are involved in other communities there is no way of proving that the members reached by this survey are entirely representative of FOSS community members in general. It may be that communities are far more focused on software development than has been demonstrated by this research. Furthermore, it is acknowledged that the data has only been collected from FOSS community members who are not opposed to filling in surveys. This of course is a potential problem for all academic research but as a person's views on surveys are not directly related to their views on the subject matter, this should not significantly distort the results.

The research has investigated communities that are involved with both support and development activities and consequently has collected data from the different types of members. The data has shown that in terms of support, problem solving is the main reason that members have for using FOSS communities, concurring with the work by Lakhani \& Wolf (2003). Interestingly however, only slightly fewer members chose providing support as a reason. This suggests two things; firstly that the majority of FOSS community members, in this type of community, perceive support as being the primary reason or function of the community. Secondly that members rank getting help from others, and giving it to others, as equally important. The moral views of Stallman (1999) therefore may be just as applicable now as they were during the early years of Free Software. Although it is possible that members who prefer to receive support rather than give it may be less likely to fill in a survey, the significant number of members who chose providing support as a reason for participating, shows that this view is common among FOSS community members. It also shows that those involved in FOSS are aware and appreciate the importance of sharing and collaboration in community systems as well as software development.

Members also saw peer review and actual software development as being of equal importance. Since peer review can be performed by member who may have little or no knowledge of software development, in the programming sense, this highlights the importance of the user in the FOSS development process and the close user-developer relationship that exists (Scacchi 2005). It also demonstrates that FOSS communities are highly involved in the development of software, even when many of the participating members are not contributing code and may not even be programmers. These contributions would instead be in the form of software testing, bug reporting and general suggestions on function and operation (Pavlicek 2000; Moody 2001; Raymond 1999). If these results are to be considered representative of FOSS communities in general, the results would suggest that only approximately $50 \%$ of member activities within the community are for reasons of software development. This supposition is however dependent on the factors of survey participation and sample set community types. 
An extremely interesting result was the apparent importance of social exchange within the communities. $70 \%$ of the surveyed members, stated that meeting and talking to people with similar interests was one of their main reasons for their participation. This made up $21 \%$ of the reasons for member participation (See Figure 2). Sagers' (2004) and O'Mahony's (2004) work would seem to fit in with these findings. However, in specific terms of support and development (See figure 4 through 6), only around $1 \%$ of members felt that social factors drove them to use support or development forums. This suggests that the social activities within the communities are not confined either to support or development activities but instead extend to broader social interest.

The second phase of the research, investigating how members use the communities, has also produced some interesting results and helped to define the different types of members that make up a community. From this sample set, the majority of members (36\%) use communities for getting support with their software and giving support to others. Logically this means that many members will login to a FOSS community website only to help others with their problems, quite possibly with no tangible benefit to themselves. This correlates with the results of the first phase, in which $25 \%$ of members listed providing support as a reason for participation (See Figure 2). A slightly smaller number of members stated that they would participate only if it was useful for them to do so, suggesting that, in terms of support, the two types of community members are those who perceive giving and taking as being equally important, and those who require some incentive or personal benefit for them to participate. Additionally, Zhang \& Storck's (2001) research into "peripheral members", supports the research's finding that approximately $23 \%$ of members will observe the community but rarely participate themselves. This too could be a matter of incentive but is a very difficult subject to research given the apparent unwillingness of the members to participate. It is quite possible that there are a great deal more members that very rarely participate in the sample communities and consequently were not reached by this survey.

The members of the community involved in development provided a much more clear-cut set of results. The majority of them (66\%) stated that they were involved in FOSS development communities both to get help with their work, and help others with theirs, again demonstrating the attitude of collaboration and team work that exists within FOSS. Only $20 \%$ of members said that they participated only to get help with their own work. This mirrors the findings from the support communities but indicates that the bi-direction collaborative aspects are more important in actual software development. Only a very small number of members participated to get involved in others projects. It is likely that these will be new members, attempting to get involved with projects for educational purposes. 


\section{Conclusions}

The presented research has extracted information about FOSS communities from the very members that they consist of. It is this unique viewpoint that has revealed the very interesting inferences that have been taken from the research findings. It has looked at the ways in which members of a FOSS community perceive the group that they are in, and has revealed some of the very specific motivational aspects involved.

Although FOSS communities are still often seen as ad-hoc and chaotic, the research has shown that it is common interest and community relations that bind these communities together, and allows them to produce both knowledge and software in such an effective fashion. The research has demonstrated that there is strong sense of sharing and collaboration within communities that support FOSS development and use. This manifests itself in two main ways, firstly in the areas of software development where code, ideas and suggestions are shared and secondly in the software support area, where information about software use is the object of transfer. It is this code and knowledge generation and transference between community members with diverse sets of expertise and backgrounds that allows FOSS communities to function so well.

\section{References}

Dibona, C., Ockham, S., Stone, M. (1999), Open Sources: Voices from the Open Source Revolution, O'Reilly \& Associates, Inc., CA, USA

Hann, I. H., (2004) "Why Developers Participate in Open Source Software Projects: And Empirical Study", Twenty-Fifth International Conference on Information Systems.

Hertel, G., Niedner, S., Herrmánn, S. (2003), "Motivation of Software Developers in Open Source Projects: An Internet-based Survey of Contributors to the Linux Kernel", Research Policy, Special Issue on Open Source Software Development, Available at: http://opensource.mit.edu/papers/hertel.pdf, Accessed (February 2004)

Krishnamurthy, S., (2002) "Cave or Community? An Empirical Examination of 100 Mature Open Source Projects", Available at http://opensource.mit.edu/, Accessed (Feb 2005)

Lakhani, K. R., Wolf, R.G. (2003), "Why Hackers Do What They Do: Understanding Motivation Effort in Free Open Source Software Projects", MIT Sloan School of Management Working paper, at: http://freesoftware.mit.edu/papers/lakhaniwolf.pdf, Accessed (February 2004) 
Lanzara, G.F., Morner, M. (2003), 'The knowledge ecology of Open-Source Software Projects', paper presented at seminar on 'ICTs in the contemporary world' at LSE Department of Information Systems on 2nd October

Moody, G. (2001), "Rebel Code: How Linus Torvalds, Linux and the Open Source Movement Are Outmastering Microsoft", The Penguin Press, England

O'Mahony, S., Ferraro, F., (2004) "Hacking Alome? The Effects of Online and Offline Participation on Open Source Community Leadership", Available at http://opensource.mit.edu/, Accessed (Dec 2004)

Oh, W., Jeon, S., (2004) "Membership Dynamics and Network Stability in the Open-Source Community: The Ising Perspective" Twenty-Fifth International Conference on Information Systems.

Pavlicek, R. C. (2000), "Embracing Insanity: Open Source Software Development", Sams Publishing, USA

Raymond, E. S, (1999), "The Magic Cauldron",

Available at: http http://www.catb.org/-esr/writings/cathedral-bazaar/magic-cauldron/ (Accessed December 2003)

Raymond, E. S, (2000), "The Cathedral and the Bazaar",

Available at: http://www.catb.org/ esr/writings/cathedral-bazaar/cathedral-bazaar/ (Accessed November 2003) and in Dibona et al (1999)

Sagers, G.W., (2004) "The Influence of Network Governance Factors on Success in Open Source Software Development Projects", Twenty-Fifth International Conference on Information Systems.

Scacchi, W., Feller, J., Fitzgerald, B., Hissam, S., Lakhani, K., (2005) "Understanding Free/Open Source Software Development Processes", Available at: (http://www.ics.uci.edu/ wscacchi/Papers/New/SPIP-FOSS-Intro-Dec2005.pdf) (Accessed December 2005)

Schofield, A., Mitra, A. (2004), "Complexities of Classifying Open Source: Developing a Framework for Categorising Open Software Development", UK Academy of Information Systems conference 2004, Glasgow, UK.

Schofield, A., Mitra, A. (2005), "Free and Open Source Software Communities as a Support Mechanism", UK Academy of Information Systems conference 2005, Newcastle, UK

Stallman, R. (1999), "The GNU Operating System and the Free Software Movement", in Dibona et al (1999).

Zhang, W. \& Storck, J, (2001) "Peripheral Members in Online Communities", Americas Conference on Information Systems, Boston, USA. 